\title{
Adrenal Lesion Core Biopsy: A New Approach and an Incidental Benefit
}

\author{
Kim Smith* \\ Radiology SA Central Districts, Australia
}

\begin{abstract}
A 79-year-old male with a history of renal cell carcinoma was referred for CT guided core biopsy of a right sided $3 \mathrm{~cm}$ adrenal lesion. A number of vulnerable anatomical structures were adjacent to the lesion, so an innovative positioning technique was used to maximize access to the lesion and minimize risk to the adjacent structures. The head down position used in this case resulted in the liver and portal vein moving superiorly, significantly reducing the likelihood of injury. This case study briefly discusses the pathologies and diagnoses of adrenal lesions and explains the value of the unique approach used.
\end{abstract}

Keywords

Adrenal, Biopsy, Vena cava

\section{Summary of the Reviewed Literature}

In a group of 87065 autopsies, 6\% revealed incidental adrenal lesions [1,2]. A wide variety of methods are currently used to assess lesions of the adrenal gland, a review of the current literature provides excellent description of these techniques.

There are well established criteria for deciding whether adrenal lesion biopsy is appropriate.

In patients with a history of a prior extra adrenal neoplasm, $50-75 \%$ of adrenal lesions seen are malignant $[3,4]$. This is in stark contrast to the statistics for lesions in patients with no prior cancer history. Incidentally found adrenal lesions in otherwise well patients are often benign adrenal adenoma [5]. Only $6 \%$ of lesions less than $4 \mathrm{~cm}$ in diameter prove neoplastic with that number increasing to $25 \%$ in lesions measuring 4-6 cm [5]. In view of these values, adrenal lesions measuring $4 \mathrm{~cm}$ or less are not typically biopsied.

In the absence of a cancer history, a non-contrast CT of the adrenal glands is the accepted practice in our organization. Benign adrenal adenoma is the most common finding [6]. Adrenal adenoma is seen as a discrete lesion of relatively uniform density with an average Houndsfield Units (HU) value of 10 or less. Lesions fitting this description are considered low risk and are managed conservatively. Lesions of higher density require more vigorous investigation, especially those which are rapidly growing or greater than 6 $\mathrm{cm}$ in diameter. In particular, lesions with irregular contours are suspicious of malignancy and can further be characterized through a multiphasic CT examination. Due to the risk of hypertensive crisis in pheochromocytoma, evaluation of the lesion prior to biopsy is advised.

\section{Equipment}

Siemens Definition 64 CT scanner installed in May 2010. A $30 \mathrm{~cm}$ transparent ruler and non-alcohol soluble marker.

A $10 \mathrm{ml}$ syringe filled with $10 \mathrm{ml}$ normal saline solution and fitted with a $23 \mathrm{~g} \times 38 \mathrm{~mm}$ needle for washing samples from the core needle. Sterile, disposable trolley drape, fenestrated drape, gloves and surgical gown. Dressing tray with gauze, $10 \mathrm{ml}$ syringe, $18 \mathrm{~g}$ blunt drawing up needle, scalpel and pathology sample pot containing formalin. $2 \%$ solution of Chlorhexidine in $70 \%$ alcohol as skin antiseptic. $15 \mathrm{ml}$ Xylocaine $1 \%$ solution administered via a $50 \mathrm{~mm} \times 22 \mathrm{~g}$ needle as local anaesthetic. $10 \mathrm{~cm} \times 17 \mathrm{~g}$ biopsy introducer needle with a $15 \mathrm{~cm} \times 18 \mathrm{~g}$ core biopsy needle inserted coaxially.

\section{Technique}

Following an explanation of the procedure to the patient, signed consent was obtained once no contraindications were established.

The patient was positioned prone on the CT table with three pillows under the pelvis and a long triangular pad under the ankles to relieve stress on the feet. Padding for the shoul-

*Corresponding author: Kim Smith, Site Senior, Radiology SA Central Districts, Australia, Tel/Fax: 84020210

Accepted: September 28, 2019

Published online: September 30, 2019

Citation: Smith K (2019) Adrenal Lesion Core Biopsy: A New Approach and an Incidental Benefit. Insights Biomed Res 3(1):72-74 
ders and chest was kept to a minimum to keep the torso in what was effectively a prone "Trendelenburg" position. A 12 $\mathrm{cm}$ CT opaque marker was positioned to the right of the spine centred at the lower costal margin. A planning dataset of 1 $\mathrm{mm}$ thick, contiguous, helically acquired images was obtained in expiration. In consultation with the radiologist the lesion identified. A needle path which avoided both lung and liver was planned and the skin was marked appropriately.

The skin surrounding the mark was washed antiseptically by a radiologist wearing radiation safety protection equipment, sterile gloves and gown. The fenestrated drape was applied and $10 \mathrm{ml}$ of local anaesthetic injected at the marked site. An incision was made and the introducer needle inserted. Imaging was used to aid manipulation of the introducer. At the abdominal wall an additional $5 \mathrm{ml}$ of Xylocaine was injected to minimise the patient's discomfort when the abdominal wall was traversed. To secure access with the core needle, the introducer was positioned to "pin" the lesion.

Three core samples were obtained, each washed from the core needle tip using a jet of saline into the pot of formalin. Minimal bleeding was observed from the site, which was managed with pressure for two minutes and the application of a $3 \mathrm{~cm} \times 6 \mathrm{~cm}$ self-adhesive non-stick dressing. The patient was rolled into a supine position on a barouche and made comfortable. Transport to the recovery ward for observation was arranged and the patient was discharged two hours later. Prior to discharge, a chest X-ray was performed to ensure there had been no injury to the adjacent diaphragm resulting in pneumothorax. During the patient's recovery stay, all observations were well within normal limits and no pain management protocol was required.

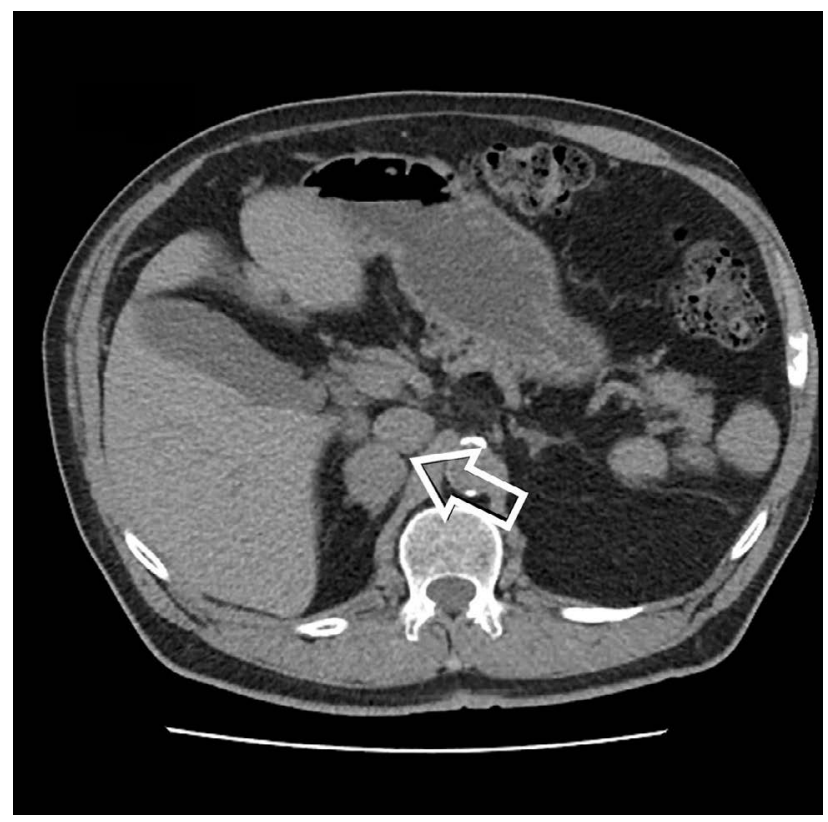

Figure 1: Previous supine diagnostic scan. White arrow indicates the proximity of the Vena Cava to the anterior surface of the adrenal mass.

\section{Discussion}

The simple but effective positioning technique used in this case has not been described in the articles reviewed, and may prove useful in other cases.

The subject of this case had his right kidney resected for renal cell carcinoma, so despite the $3 \mathrm{~cm}$ diameter of the adrenal gland lesion, a biopsy was appropriate. The referring physician did not have concerns that the lesion was a pheochromocytoma, so the requested biopsy proceeded.

Anatomically the right adrenal gland is adjacent to the inferior part of the right lung posteriorly, liver laterally, spine medially and inferior vena cava anteriorly. This position limits the opportunities available for low risk access for percutaneous biopsy. Previous diagnostic CT examination of this patient also showed the portal vein abutting the anterior surface of the lesion (Figure 1). Following discussion with the performing radiologist, the patient was positioned prone with three pillows elevating the pelvis. This resulted in a "head down" angle at the biopsy site which was designed to afford access to the lesion while avoiding the posterior diaphragm. Expiratory arrested respiration aided in this effort during imaging and needle manipulation.

In addition to the risk of injury while approaching the biopsy site, there is potential for injury if the needle traverses the lesion entirely. The head down position used in this case resulted in the liver and portal vein moving superiorly, significantly reducing the chance of injury. As an incidental benefit, the inferior vena cava was seen to be less than half the diameter seen in the previous supine imaging due to a drop in the abdominal venous pressure in this position (Figure 2 and Figure 3). The post procedure chest X-ray (Figure 4) showed

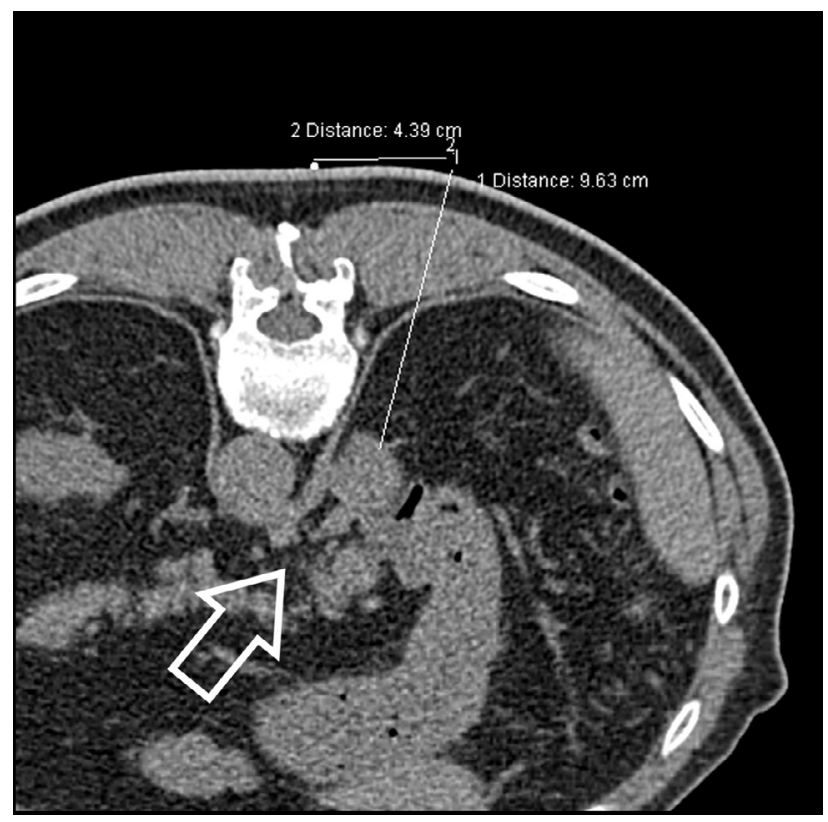

Figure 2: Planning scan. Needle approach is measured and skin marked, white arrow indicates the region previously occupied by distended Vena Cava. 


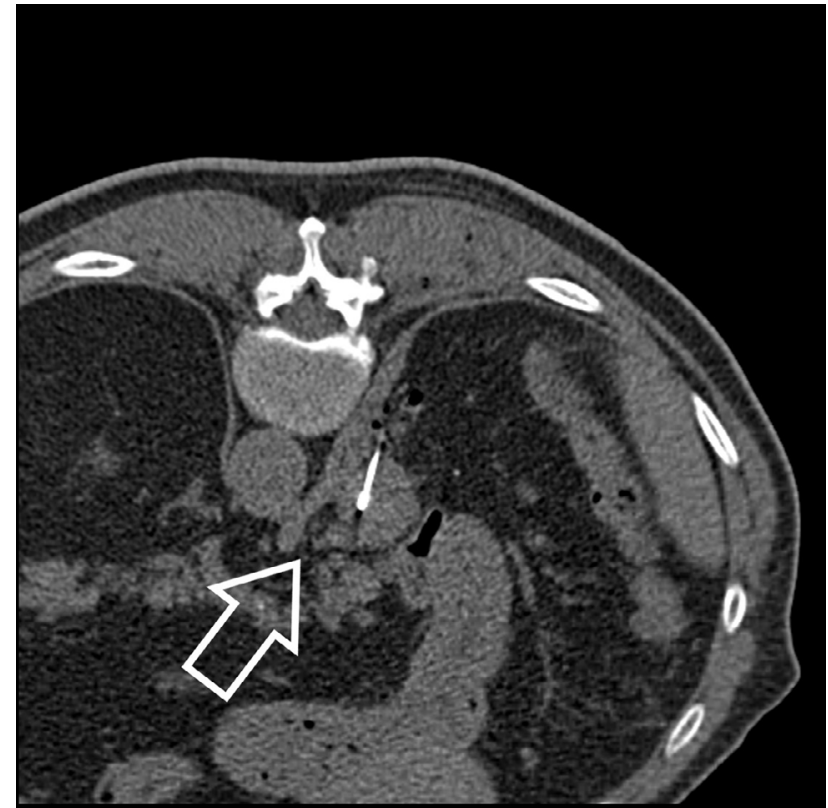

Figure 3: Procedure scan. White arrow indicates region of previously distended Vena Cava and the coring needle is seen in the adrenal mass.

no pneumothorax to indicate diaphragmatic or pleural injury.

The pathology report of the tissue sampled was in keeping with the current literature. Clear cell proliferation of metastatic renal cell carcinoma, World Health Organisation Grade 1 was found.

\section{Conclusion}

The criteria for adrenal lesion fine needle aspiration (FNA) or core biopsy are well established and were clearly met in this case. A beneficial result with minimal risk to the patient was obtained through careful positioning and technique. The incidental benefit of the position used contributed to the safety of the procedure by minimizing the diameter of the adjacent Vena Cava. This position was not found in a search of previously published material and may be of significant value in future cases.

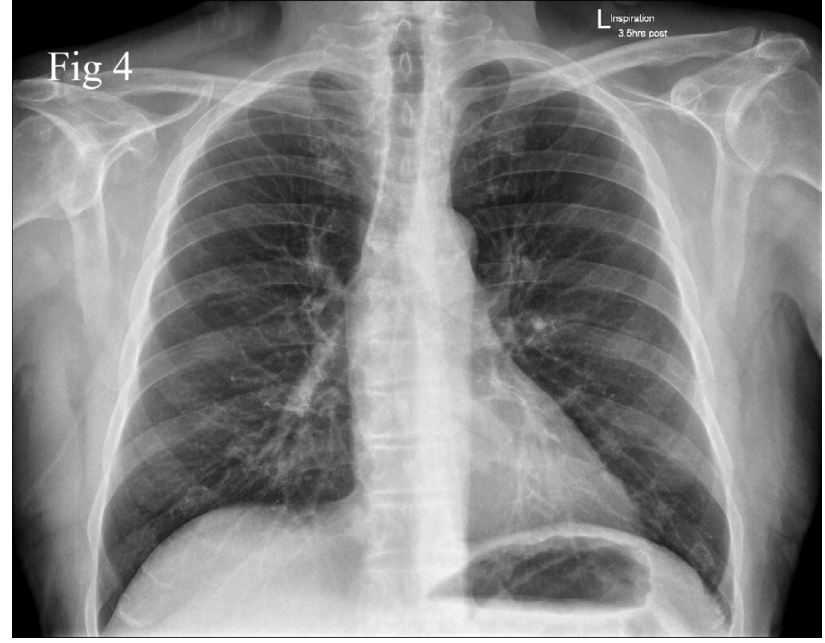

Figure 4: Post procedure chest radiograph. No evidence of injury.

\section{References}

1. Young WF (2007) Clinical practice. The incidentally discovered adrenal mass. N Engl J Med 356: 601-610.

2. Kloos RT, Gross MD, Francis IR, et al. (1995) Incidentally discovered adrenal masses. Endocr Rev 16: 460-484.

3. Lenert JT, Barnett CC, Kudelka AP, et al. (2001) Evaluation and surgical resection of adrenal masses in patients with a history of extra-adrenal malignancy. Surgery 130: 10601067.

4. Frilling A, Tecklenborg K, Weber F, et al. (2004) Importance of adrenal incidentaloma in patients with a history of malignancy. Surgery 136: 1289-1296.

5. Alexandraki KI, Grossman AB (2008) Adrenal incidentalomas: "The rule of four." Clin Med 8: 201-214.

6. Dinnes J, Bancos I, Di Ruffano LF, et al. (2016) Management of endocrine disease: Imaging for the diagnosis of malignancy in incidentally discovered adrenal masses: A systematic review and meta-analysis. Eur J Endocrinol 175: 51-64.

DOI: $10.36959 / 584 / 450$ 